\title{
Development of an approach to assess critical doses and dose rates for cultivated plants
}

\author{
A.A. Oudalova, L.N. Ulyanenko and S.A. Geras'kin \\ Russian Institute of Agricultural Radiology and Agroecology, 249032 Obninsk, Russia
}

\begin{abstract}
The aim of this work is to develop methods for an assessment of critical doses and dose rates that can result in significant radiation-induced effects in agrocenoses. This is realized on an example of cultivated plants which are one of the main components of agroecosystems. Available information on dose dependences in such umbrella endpoints as reproduction, survival, morbidity, alterations in morphological and biochemical processes, genetic effects in crops, vegetables, fruit trees, etc are gathered from papers issued mainly in Russian scientific press during last 50 years. Data are maintained as database in MS Access that contains about 10000 records at the moment. As critical exposure values, doses are considered producing $50 \%$ changes of biological effect at acute impact $\left(\mathrm{ED}_{50}\right)$, or dose rates resulting in $10 \%$ changes at chronic exposure of plants $\left(\mathrm{EDR}_{10}\right)$. The critical doses and dose rates for agrocenosis estimated from available information on reproduction and survival are presented here. Primary data are assesed for their quality according to several criteria. Three models (linear, logariphmic, logistic) for dose-effect relationship are tested for an applicability to fit a dose-effect dependence taking account of their goodness-of-fit and robustness of $\mathrm{ED}_{50}$ and $\mathrm{EDR}_{10}$ estimates.
\end{abstract}

\section{INTRODUCTION}

In recent years many efforts have been undertaken to develop a system of radiation protection for nonhuman biota [1,2]. Agrarian ecosystems are of special concern from the viewpoint of establishing safe levels of radiation impact on the environment because a) their contamination can affect human health via radionuclide uptake with food, and b) agroecosystems are ones of the most sensitive biocenoses to a number of environmental impacts including ionising radiation [3]. While the existing system of radiation standards [4] is effective in providing radiation protection of human and restricts radionuclides content in food chains, there are no guidelines on setting any limitations to directly protect agrarian ecosystems from negative effect of radiation or to demonstrate their protection at radiation levels safe for human. The aim of this work is to develop methods for an assessment of critical doses and dose rates which violation can result in significant radiation-induced effects in agrocenoses. This is realized on an example of cultivated plants as the main component of agroecosystems. Cultured plants not only contribute essentially to food production but also fulfil an important ecological function in agrarian biocenosis.

\section{MATERIALS AND METHODS}

\subsection{Database}

Available information on radiation-induced effects in cultured plants are being gathered from papers issued mainly in Russian scientific press during last 50 years; the work is ongoing. Data are maintained as database in MS Access that now contains about 3000 records from 175 original sources (161 in Russian). There are available about 10000 entries which are pairs of "exposure level - biological effect" data accompanied with information on experimental design, species and its life stage, exposure parameters and conditions. Quantitative data are collected for about 60 species of cultivated plants. Most 
information found concern radiation effects in crops, vegetables and roots, as well as legumes $-38 \%$, $21 \%$ and $18 \%$ of all entries, correspondingly.

Biological responses are grouped under umbrella endpoints of reproduction, survival, morbidity, alterations in morphological and biochemical processes, and (cyto)genetic effects. Only findings from an analysis of reproduction and survival endpoints at acute and chronic irradiation of plants during vegetation are presented in this paper. Reproduction endpoint pools traits of biological productivity (seed and straw mass, dry and wet biomass, number of plants per $1 \dot{\mathrm{m}}^{2}$, etc) and reproductive potential (portion of fertile pollen seeds, fertility/sterility, number or portion of male and female flowers, etc). The survival indexes are germination ability and rate (in field or in laboratory), survival of germinated seeds or plants by certain time/day, etc.

\subsection{Dose (rate) - effect models}

Each dataset was substituted for regression analysis to reconstruct the dose(rate) - effect relationship and then estimate the critical exposure values that were defined the same way as in $[5,6]$. Thus, the dose giving $50 \%$ change in observed effect $\left(\mathrm{ED}_{50}\right)$ in comparison to control is considered as the critical dose for acute exposure, and the critical dose rate for chronic exposure is defined as the dose rate resulting in $10 \%$ change of observed effect $\left(\mathrm{EDR}_{10}\right)$. Three models were tried and tested for an applicability to fit the dose(rate)-effect dependences taking account of their goodness-of-fit and robustness of $\mathrm{ED}_{50}$ and $\mathrm{EDR}_{10}$ estimates:

$$
\begin{aligned}
\text { A. linear model } & y=a+b \cdot x, \\
\text { B. logarithmic model } & y=a+b \cdot \ln (x), \\
\text { C. logistic model } & y=c\left(1-\frac{x^{a}}{x^{a}+b^{a}}\right) .
\end{aligned}
$$

For the logarithmic model spontaneous effect data at $x=0$ can not be used because the logarithm argument must be over zero $(x>0)$.

\subsection{Data quality assessment}

At the $\mathrm{ED}_{50} / \mathrm{EDR}_{10}$ computation, primary data were assesed for their quality according to several rules, some of them were similar to those proposed in $[5,6]$ for data quality assurance:

Rule 1. Volume of dataset $n$ must be enough for regression analysis with a given model, i.e. degrees of freedom $d f$ should be $>0$, where $d f=n-n p-1, n p$ - the number of free parameters in a model.

Rule 2. The variation of effect with dose or dose rate should be monotonous and consistent with the current knowledge in radiobiology.

Rule 3. Regression should be significant at least at $p<0.10$.

Rule 4. At least one effect observation $y_{i}$ should be located within 10 to $90 \%$ of the effect variation range $\left[y_{x=0}, y_{x=}\right)$;

Rule 5. The estimated critical ecotoxicity value should be within the dose(rate) range studied: $x_{\min }<$ $\mathrm{ED}_{50}<x_{\max }$ or $x_{\min }<\mathrm{EDR}_{10}<x_{\max }$.

\section{RESULTS AND DISCUSSION}

\subsection{Datasets of good quality}

In total there were available 498 datasets on the endpoints of reproduction and survival. Over $80 \%$ of them did not meet the requirements of the five rules to the data quality (Table 1). In general, the number of proper datasets depends on the dose-effect relationship chosen because of different degrees of freedom and varying goodness-of-data fitting. At the application of the linear and logistic models less 
Table 1. Quality assessment for data on reproduction and survival of cultured plants at acute and chronic exposure during vegetation.

\begin{tabular}{|l|c|c|c|c|c|c|c|c|c|c|c|c|c|}
\hline & \multicolumn{9}{|c|}{ Acute exposure (330 datasets) } & \multicolumn{7}{c|}{ Chronic exposure (168 datasets) } \\
\cline { 2 - 15 } & \multicolumn{1}{|c|}{ Number of datasets meeting requirements } \\
\hline Rule & 1 & 2 & 3 & 4 & 5 & All rules & 1 & 2 & 3 & 4 & 5 & All rules \\
\hline Model & & & & & & & & & & & & \\
\hline Linear $(n p=2)$ & 134 & 119 & 89 & 119 & 71 & 63 & 84 & 51 & 20 & 69 & 62 & 18 \\
\hline Logarithmic $(n p=2)$ & 100 & 120 & 50 & 119 & 62 & 39 & 72 & 51 & 17 & 66 & 54 & 13 \\
\hline Logistic $(n p=3)$ & 96 & 113 & 69 & 114 & 82 & 55 & 63 & 51 & 26 & 69 & 60 & 27 \\
\hline
\end{tabular}
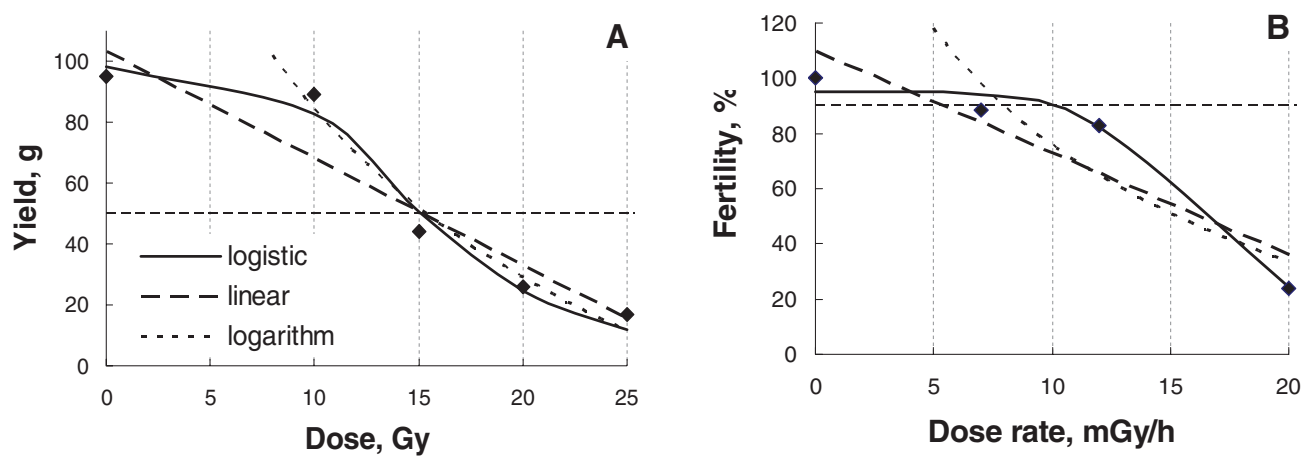

Figure 1. Approximation of data with three models and estimation of the critical dose (A) and dose rate (B) A Yield of grains from corn plants exposed on the 54 day of growing [7]; $\mathrm{ED}_{50}=15.9,15.8$, and $15.5 \mathrm{~Gy}$ from linear, logarithmic and logistic models, correspondingly. B - Fertility of the main ear of wheat plants exposed in $\gamma$-field from 2-week seedlings to harvesting $16 \mathrm{~h}$ per day [8]; $\mathrm{EDR}_{10}=5.40,7.92$, and $10.1 \mathrm{mGy} / \mathrm{h}$

data were rejected then with the logarithmic model. Many original studies give only two observations typically referring to the control and impacted groups. This is why up to $70 \%$ of the datasets are rejected by Rule 1 . Among the rest data, many datasets do not meet requirements of sufficient quality of data fitting, i.e. regression significance (Rule 3). Also, Rule 5 appeared to be quite restricting for acute exposure data, and Rules 2 - for chronic exposure (likely because of observations of stimulating effect at low dose rates).

\subsection{Critical ecotoxicity values}

Figure 1 illustrates an approximation of data on reproduction endpoint by three dose(rate)-effect models and estimates of the critical dose and dose rate at acute and chronic exposure, correspondingly. Calculated critical ecotoxicity values are presented in Table 2 (acute exposure) and Table 3 (chronic exposure) as average $\mathrm{ED}_{50}\left(\mathrm{EDR}_{10}\right)$ with standard error and range of variation for a given species.

Data of good quality on survival are scarce both at acute and chronic exposure. Critical ecotoxicity values are obtained only from one or two datasets for every species. For few species, where data are available for both endpoints considered (corn, rice, soya for acute, wheat and barley for chronic exposure), $\mathrm{ED}_{50}$ and $\mathrm{EDR}_{10}$ are lower when estimated from reproduction (Table 2 and 3). Thus, reproduction seems to be more radiosensitive criteria than survival, which is in line with our previous findings [9] and other studies [11, 12].

It is obvious that critical exposure values depend on the model used. An analysis of the data available shows that at acute exposure the differences in $\mathrm{ED}_{50}$ obtained from three models appeared to be insignificant $(p>0.05)$ for all species, both for reproduction and survival endpoints. 
Table 2. Critical doses for species of cultivated plants after acute exposure.

\begin{tabular}{|c|c|c|c|c|c|c|c|c|c|}
\hline \multirow{3}{*}{ Species } & \multicolumn{3}{|c|}{ Linear } & \multicolumn{3}{|c|}{ Logarithm } & \multicolumn{3}{|c|}{ Logistic } \\
\hline & \multirow[t]{2}{*}{$\mathrm{N}$} & \multicolumn{2}{|c|}{$\mathrm{ED}_{50}, \mathrm{~Gy}$} & \multirow[t]{2}{*}{$\mathrm{N}$} & \multicolumn{2}{|c|}{$\mathrm{ED}_{50}, \mathrm{~Gy}$} & \multirow[t]{2}{*}{$\mathrm{N}$} & \multicolumn{2}{|c|}{$\mathrm{ED}_{50}, \mathrm{~Gy}$} \\
\hline & & mean \pm se & $\min -\max$ & & mean \pm se & $\min -\max$ & & mean \pm se & $\min -\max$ \\
\hline \multicolumn{10}{|c|}{ Reproduction } \\
\hline Corn & 5 & $11.9 \pm 1.1$ & $10.1-15.9$ & 4 & $11.8 \pm 1.6$ & $8.5-15.8$ & 6 & $13.4 \pm 2.2$ & $8.1-23.4$ \\
\hline Oats & 5 & $20.9 \pm 5.5$ & $10.2-34.5$ & 5 & $13.9 \pm 2.2$ & $9.4-19.8$ & 6 & $15.6 \pm 2.4$ & $9.4-21.5$ \\
\hline Wheat & 17 & $12.5 \pm 1.5$ & $7.7-27.4$ & 6 & $11.4 \pm 1.5$ & $7.1-15.8$ & 11 & $9.9 \pm 1.4$ & $3.0-17.4$ \\
\hline Rice & 2 & $219.3 \pm 8.6$ & $211-228$ & 1 & 115.3 & & 2 & $118 \pm 19.8$ & 98.7-138 \\
\hline Barley & 6 & $9.9 \pm 1.2$ & $5.3-14.1$ & 5 & $7.5 \pm 1.5$ & $5.1-13.0$ & 6 & $9.9 \pm 2.3$ & $4.8-19.0$ \\
\hline Soya & 4 & $17.7 \pm 2.6$ & $11.7-23.3$ & 4 & $14.3 \pm 2.5$ & $10.3-20.9$ & 4 & $14.5 \pm 2.4$ & $9.8-19.8$ \\
\hline Potato & 7 & $54.1 \pm 18.6$ & $22.5-154$ & 3 & $22.4 \pm 3.8$ & $15.2-28.3$ & 4 & $39.6 \pm 17.3$ & $16.3-28.4$ \\
\hline Strawberry & 1 & 101.5 & & 1 & 56.5 & & 1 & 63.4 & \\
\hline Clover & 3 & $222 \pm 64.2$ & $137-348$ & 3 & $163 \pm 28.9$ & $123-219$ & 3 & $163 \pm 34.5$ & $116-230$ \\
\hline Fescue & 2 & $33.6 \pm 4.0$ & $29.6-37.7$ & 1 & 26.8 & & 3 & $33.0 \pm 3.9$ & $25.3-37.6$ \\
\hline Ryegrass & 2 & $21.3 \pm 2.1$ & $19.2-23.5$ & 1 & 20.6 & & 1 & 19.6 & \\
\hline Cotton & 1 & 66.7 & & 1 & 63.1 & & 1 & 60.4 & \\
\hline \multicolumn{10}{|c|}{ Survival } \\
\hline Corn & & & & & & & 1 & 22,9 & \\
\hline Rice & 1 & 244 & & 1 & 216.8 & & 1 & 232 & \\
\hline Beans & 2 & $1.9 \pm 0.4$ & $1.48-2.33$ & 1 & 1.2 & & 2 & $1.7 \pm 0.6$ & $1.12-2.24$ \\
\hline Soya & 2 & $32.8 \pm 1.7$ & $31.1-34.5$ & 2 & $29.8 \pm 1.6$ & $28.2-31.3$ & 2 & $33.5 \pm 0.7$ & $32.8-34.3$ \\
\hline Agropyron & 1 & 21.8 & & & & & 1 & 20.4 & \\
\hline Tobacco plant & 2 & $0.02 \pm 0.00$ & $0.021-0.025$ & & & & & & \\
\hline
\end{tabular}

$\mathrm{N}$ - number of datasets.

In the case of chronic irradiation, the critical dose rates $\mathrm{EDR}_{10}$ obtained with the logistic model are always higher than that from the linear one (Table 3), sometimes up to 2 orders of magnitude. This is because a logistic curve changes very slowly at low dose rates, and thus reaches the $10 \%$ decrease from the control level at higher values of dose rate (Fig. 1B). But variance of the 'logistic' $\mathrm{EDR}_{10}$ values estimated from different datasets for the same species is so high that the average EDR $_{10}$ does not differ from 0 for most cases (Table 3). This makes differences with the 'linear' $\mathrm{EDR}_{10}$ values insignificant for all species. Such a high variability is bound to a lack of good quality effect data, which is recognized for chronic radiation exposure of biota in general [13] and agricultural plants, in particular [9]. Also, the original studies differ very much in methods and study design, dosemetric information is often incomplete, studied ranges of dose rates poorly overlap.

\subsection{Comparison to previous findings}

In our previous contribution [9], the critical doses to species of cultivated plants were estimated from the linear dose-effect relationship. Original effect data were transformed in relative units as $\%$ of corresponding controls, pooled together to form arrays under every specific endpoint, and submitted for regression analysis. That way of data handling does not require rejecting datasets that violate the rules of data quality and, thus, allows using much more data for calculations than the approach used in present study. Indeed, the pooled arrays included together nearly 600 pairs of data on reproduction and survival [9], while 81 datasets meeting the five rules requirements for linear model in this work (Table 1) contain only about 370 paired data entries even despite the database has been enlarged from 7000 to 10000 entries.

Findings from two approaches (pooled arrays and individual datasets) are compared in Table 4. Critical doses or dose rates calculated from reproduction data are presented when there are estimates for the same species. To ensure some confidence in critical doses estimated from pooled data, two requirements were fulfilled in [9]: 1) regression significance of at least 10\%, 2) a minimal number of 
Table 3. Critical dose rates for species of cultivated plants after chronic exposure.

\begin{tabular}{|c|c|c|c|c|c|c|c|c|c|}
\hline \multirow{3}{*}{ Species } & \multicolumn{3}{|c|}{ Linear } & \multicolumn{3}{|c|}{ Logarithm } & \multicolumn{3}{|c|}{ Logistic } \\
\hline & \multirow[t]{2}{*}{$\mathrm{N}$} & \multicolumn{2}{|c|}{$\mathrm{EDR}_{10}, \mathrm{mGy} / \mathrm{h}$} & \multirow[t]{2}{*}{$\mathrm{N}$} & \multicolumn{2}{|c|}{$\mathrm{EDR}_{10}, \mathrm{mGy} / \mathrm{h}$} & \multirow[t]{2}{*}{$\mathrm{N}$} & \multicolumn{2}{|c|}{$\mathrm{EDR}_{10}, \mathrm{mGy} / \mathrm{h}$} \\
\hline & & mean \pm se & $\min -\max$ & & mean \pm se & $\min -\max$ & & mean \pm se & $\min -\max$ \\
\hline \multicolumn{10}{|c|}{ Reproduction } \\
\hline Wheat & 7 & $6.5 \pm 0.8$ & $5.4-11.2$ & 2 & $9.7 \pm 1.5$ & $7.9-11.0$ & 9 & $230 \pm 218$ & $9.8-1977$ \\
\hline Barley & 2 & $139 \pm 12.7$ & $126-152$ & 1 & 3.8 & & 3 & $120 \pm 58.2$ & $4.0-185$ \\
\hline Bean & 3 & $8.0 \pm 1.7$ & $7.4-11.1$ & 2 & $6.7 \pm 0.6$ & $6.1-7.3$ & 3 & $16.0 \pm 6.1$ & $9.0-28.1$ \\
\hline Peas & 3 & $523 \pm 430$ & $38-1380$ & 1 & 101.8 & & 3 & $1020 \pm 775$ & $129-2567$ \\
\hline Currant & 1 & 29.2 & & 1 & 5.5 & & & & \\
\hline Lettuce & 1 & 18.5 & & 1 & 4.7 & & 2 & $2030 \pm 2000$ & $27.8-4030$ \\
\hline Tomato plant & & & & & & & 1 & 6120 & \\
\hline Tobacco plant & 1 & 4.7 & & 1 & 3.7 & & 1 & 8.5 & \\
\hline \multicolumn{10}{|c|}{ Survival } \\
\hline Wheat & & & & 1 & 3640 & & 1 & 3660 & \\
\hline Barley & & & & & & & 1 & 2150 & \\
\hline Lettuce & & & & 1 & 5750 & & 1 & 5760 & \\
\hline Tomato plant & & & & 1 & 7030 & & 1 & 7090 & \\
\hline Clover & & & & 1 & 12740 & & 1 & 12800 & \\
\hline
\end{tabular}

Table 4. Critical exposure values with 95\% confidence intervals within brackets obtained from pooled [9] and individual (this paper) datasets for reproduction endpoint.

\begin{tabular}{|c|c|c|c|}
\hline Dataset & Pooled & \multicolumn{2}{|c|}{ Individual } \\
\hline Model & linear & linear & logistic \\
\hline & \multicolumn{3}{|c|}{$\mathrm{ED}_{50}, \mathrm{~Gy}$ (acute exposure) } \\
\hline Oats & $27.8(24.4 \div 32.5)$ & $20.9(5.6 \div 36.1)$ & $15.6(9.4 \div 21.7)$ \\
\hline Wheat & $18.3(16.4 \div 20.4)$ & $12.5(9.2 \div 15.7)$ & $9.9(6.7 \div 13.1)$ \\
\hline Barley & $15.6(14.6 \div 16.6)$ & $9.9(6.7 \div 13.1)$ & $9.9(4.0 \div 15.7)$ \\
\hline Potato & $45.8(37.8 \div 42.7)$ & $54.1(8.6 \div 99.6)$ & $39.6(0 \div 94.5)$ \\
\hline & \multicolumn{3}{|c|}{$\mathrm{EDR}_{10}, \mathrm{mGy} / \mathrm{h}$ (chronic exposure) } \\
\hline Wheat & $6.8(3.3 \div 10.0)$ & $6.5(4.6 \div 8.4)$ & $230(0 \div 734)$ \\
\hline Barley & $227(173 \div 311)$ & & $120.1(0 \div 371)$ \\
\hline
\end{tabular}

datasets $\mathrm{N} \geq 10$ for single species. In present study it is considered that averaged values for $\mathrm{ED}_{50}$ and $\mathrm{EDR}_{10}$ are not safe enough if they are based on less than 3 datasets $(\mathrm{N}<3)$. From Table 4 , if the linear approximation is applied, there are no significant differences between critical exposure values obtained from pooled and individual datasets for all species except $\mathrm{ED}_{50}$ for barley. The approximation of data after acute exposure with the logistic function gives typically the lower estimates than in the first approach, and this difference is significant for oats and wheat. However, in the case of chronic exposure variance of $\mathrm{EDR}_{10}$ calculated from a logistic dependence is extremely high so that $\mathrm{EDR}_{10}$ does not differ from 0 . At the same time, the linear extrapolation gives reasonable values from both approaches. Their comparison is possible only for one species - wheat, and the critical dose rates from pooled and individual datasets are nearly equal.

\section{CONCLUSIONS}

At development of a methodology to assess critical or no-effect doses for biota as a whole or for particular groups of flora and fauna, for example, vertebrates, invertebrates and plants in [14] or domesticated plants in this work, there are many questions to be answered. It is commonly agreed that protection should be provided not for individuals but for populations and species to preserve biodiversity in ecosystems. Trying to reveal biological effect in species most suitable for verification of radiation safety standards, we have found that traits of productivity and reproduction success in cultivated plants 
appear to be more sensitive to radiation than survival endpoint. An adaptation of the ERA methodology for the environment radiation protection $[5,6,14]$ is a promising idea in our opinion, but some of its key techniques need a further testing. Thus, it is important to find an effective way of data treatment to obtain reliable estimates. The present study gives preliminary findings contributing to development of criteria to assure quality of input data and justify a dose-effect dependence shape for the environmentally relevant effects. In particular, it shows that an absence of practice to apply standard ecotoxicity tests in radiation toxicology leads to an inadmissible waste of experimental data ( $80 \%$ and more) when the ERA approach is followed. At acute exposures, the proposed approach works well, and critical doses obtained from different models are comparable. In the most important from the viewpoint of the environment protection case of chronic exposure, however, conclusions made from different approaches to data treatment can differ significantly. At this, critical dose rates estimated from the linear model seem to be less scattered and better defined than at the logistic model utilization. However, a lack of proper data makes any differences insignificant. It is obvious that a further data gathering as well as development and testing of techniques for their treatment to get justified estimates of critical doses and dose rates for biota are needed.

\section{Acknowledgments}

The work is supported by RFBR project ź 11-08-00430.

\section{References}

[1] ICRP Publication 91. A framework for assessing the impact of ionising radiation on non-human species (Pergamon Press, Oxford, 2003).

[2] ICRP Publication 108. Environmental protection: the concept and use of reference animals and plants (Pergamon Press, Oxford, 2009).

[3] Agricultural radioecology, edited by Alexakhin R.M., Korneev N.A. (Ecology, Moscow, 1991).

[4] ICRP Publication 103. 2007 recommendations of the International Commission on Radiological Protection (Pergamon Press, Oxford, 2007).

[5] Garnier-Laplace J., Della-Vedova C., Gilbin R., et al., Environ. Sci.Technol. 40 (2006) 6498-6505.

[6] Garnier-Laplace J., Copplestone D., Gilbin R., et al., J. Environ. Radioact. 99 (2008) 1474-1483.

[7] Siemer E.G., Constantin M.J., Killion D.D., In Survival of Food Crops and Livestock in the Event of Nuclear War. Edited by D.W. Bensen, A.H. Sparrow (U.S. Atomic Energy Comission Office of Information Service, N.Y., 1971) pp. 287-305.

[8] Zolotova A.A. and Shcherbakov V.K., Radiobiology. 27 (1987) 62-68 (in Russian).

[9] Oudalova A.A., Ulyanenko L.N., Alexakhin R.M., et al., Radiat.Biol.Radioecol. 50 (2010) 572581(in Russian).

[10] Oudalova A., Ulyanenko L., Geras'kin S., Filipas A., Assessment of critical doses for reproduction and survival of cultivated plants. In Proceedings of the Third European IRPA Congress, Helsinki, Finland, 14-18 June 2010 (http://www.irpa2010europe.com/ proceedings/P09.htm)

[11] Effects on non-human biota. Annex C to UNSCEAR 2008 Report to the General Assembly (United Nations, New York, 2008)

[12] Geras'kin S.A., Fesenko S.V., Alexakhin R.M. // Environ. Intern. 80 (2008) 59-74.

[13] Copplestone D., Hingston J., Real A., J. Environ. Radioact. 99 (2008) 1456-1463.

[14] Andersson P., Garnier-Laplace J., Beresford N., et al., J. Environ. Radioact. 100 (2009) 11001108. 